Please cite as Tan et al. Synthetic Metals 161, 2249 (2011)

\title{
Field effect in chemical vapour deposited graphene incorporating a polymeric gate dielectric
}

\author{
Y. Y. Tan*, L. W. Tan, K. D. G. I Jayawardena, J.V. Anguita, J. D. Carey and S. R. P. Silva \\ Nano-Electronics Centre, Advanced Technology Institute, \\ University of Surrey, Guildford GU2 7XH, United Kingdom
}

We have investigated the room temperature long channel field effect characteristics of a single graphene layer transistor incorporating a poly-4-vinyl-phenol (PVP) organic insulating layer, as an alternative to conventional oxide gate dielectric materials. High purity copper foils were used in the chemical vapour growth of the graphene layer and visible Raman analysis confirmed the presence of a high quality mono-layer carbon film. Using a channel length of $50 \mu \mathrm{m}$, a field effect hole mobility of $37 \mathrm{~cm}^{2} / \mathrm{Vs}$ was calculated, which demonstrates the possibility of an all carbon graphene based large area transistor with carrier mobilities above those found in conventional long channel all organic electronic transistors.

Keywords: Chemical vapor deposition; graphene; transistor; polymeric gate

Email: *y.tan@surrey.ac.uk Tel/Fax: +4414839866/+4414830800

University of Surrey, Guildford, Surrey, GU2 7XH, U.K. 
Please cite as Tan et al. Synthetic Metals 161, 2249 (2011)

\section{Introduction}

Graphene exhibits some remarkable electronic properties brought about by the presence of an unique $\pi$ electron system. The low energy linear dispersion relation associated with massless electrons (Dirac fermions) gives a zero bandgap and a low energy linear density of states [1]. With high room temperature carrier mobilities of several thousand $\mathrm{cm}^{2} / \mathrm{Vs}$ readily possible, graphene based nanoelectronics is an extremely active area of research [2]. As single layer graphene does not possess a bandgap, both electron and hole conduction is possible and the electrical characteristics of graphene are often described in terms of a V-shaped characteristic of current (conductivity) with gate voltage $\left(\mathrm{I}_{\mathrm{D}}-\mathrm{V}_{\mathrm{G}}\right)$. The presence of adsorbed species, which act as doping and/or scattering centres, may result in a rigid shift and rounding of the characteristic at low carrier densities [3]. Indeed, there is currently considerable research being undertaken into how the associated processing and patterning of graphene devices may adversely affect the electrical properties. However, the room temperature mobility of graphene, even after processing, is higher than that found in organic electronic materials and opens up the possibility of large area organic electronics incorporating graphene as the active channel material. From a technological point of view, being able to produce the active layer in a roll-to-roll process is highly attractive and as such reports that graphene can now be produced using chemical vapour deposition (CVD) on metal foils and roll-to-roll processing is a significant step in this regard [4, 5]. Already a 30 inch graphene layer with high optical transmission and low sheet resistance has been demonstrated using a roll to roll growth CVD method [5]. With these technological goals in mind, we report the electrical characteristics of CVD single layer graphene grown on high purity copper foil and then transferred onto poly-4-vinyl-phenol (PVP). PVP was chosen as it is one of the more commonly investigated gate dielectrics in organic FETs because it exhibits good insulation with low leakage current density and is suitable for a low cost all-solution and print fabrication process 
Please cite as Tan et al. Synthetic Metals 161, 2249 (2011)

$[6,7,8]$. Furthermore, instead of relying on complicated and expensive e-beam lithography as reported in most published data, we fabricated long channel length $(\mathrm{L} \sim 50 \mu \mathrm{m})$ bottom electrode contact FET structures to demonstrate the ease and potential for mass fabrication. We selected the bottom contact electrode topology for our investigation into the PVP gate dielectric FET performance as this electrode topology has been identified as an important route in organic FET research $[9,10]$. Our work demonstrates that an all carbon transistor with graphene as the active layer having a long channel length is possible and opens up a competitor technology in the field of hybrid graphene-organic large area electronics.

\section{Experimental}

Copper foils (> 99\% purity, mechanically polished $25 \mu \mathrm{m}$ thick) were cut into $~ 10 \mathrm{~mm}$ strips in length and cleaned in a standard three stage clean (acetone, 2-propanol and methanol). The foils were then placed in the centre of a quartz furnace and heated up to $1000^{\circ} \mathrm{C}$ under an atmosphere of $\mathrm{H}_{2}(100 \mathrm{sccm}$ flow rate) for 30 minutes. Methane $(150 \mathrm{sccm})$ was then introduced for 45 minutes while the furnace remained at $1000^{\circ} \mathrm{C}$ and after a given time the system was cooled down to $300^{\circ} \mathrm{C}$. Below $300^{\circ} \mathrm{C}$ the flow of methane was stopped and the sample was then cooled down to room temperature under vacuum. PMMA (Mw $950 \mathrm{~K}, 10 \% \mathrm{wt})$ dissolved in chlorobenzene, was spin-cast onto the sample's surface and hardened by heating at $180^{\circ} \mathrm{C}$ for 2 minutes. The sample's $\mathrm{Cu}$ support was then removed using an iron nitrate solution. The graphene/PMMA was dried, cleaned and transferred onto a $\mathrm{SiO}_{2}$ before dissolving the PMMA under a slow flow of acetone.

To fabricate a bottom gate FET with $\mathrm{SiO}_{2}$ as gate dielectrics, the bottom contact Si backgate graphene FET was produced by first evaporating $\mathrm{Ag}$ (100 nm thick) onto $\mathrm{SiO}_{2}(300 \mathrm{~nm}$ thick) on silicon substrate as the source and drain electrodes ( $\mathrm{L} \sim 50 \mu \mathrm{m}, \mathrm{W} \sim 1000 \mu \mathrm{m})$. Next, a 
Please cite as Tan et al. Synthetic Metals 161, 2249 (2011)

graphene film was transferred using the PMMA technique described previously. Electrical I-V measurements were performed using a combined Kiethley 2400 with 238 source meter. The drain voltage was set at $500 \mathrm{mV}$, while the gate voltage was being swept. In general the $\mathrm{I}_{\mathrm{DS}} / \mathrm{V}_{\mathrm{GS}}$ were recorded under room temperature in ambient atmosphere unless otherwise specified. For the case of top contact topology, Ag was evaporated after the graphene was transferred onto the $\mathrm{SiO}_{2}$.

Next the fabrication and measurements of FET devices with PVP as polymeric gate dielectrics follows as below. The PVP solution was first prepared by dissolving PVP in propylene glycol monomethyl ether acetate (PGMEA), after which polymelamine-co-formaldehyde (PMF) was added as a crosslinking agent to the solution. Evaporated Ag (100 nm thick) on Corning glass, acting as the back gate electrode was first deposited before spin-coating the PVP solution followed by a thermal curing for three minutes at $120^{\circ} \mathrm{C}$. The PVP film was then UV photo-cured for three minutes for further hardening. The thickness of the PVP film was measured by AFM (Veeco Dimension) to be $1 \mu \mathrm{m}$ thick. Ag was again evaporated onto the cured PVP film as source and drain electrodes (i.e. bottom contact electrode topology). Finally, graphene was transferred over to complete the back-gate FET device fabrication using the same PMMA method. It was not possible to perform Raman measurements on graphene transferred on to PVP as this structure is transparent. Electrical I-V measurements were again performed with drain voltage set at $0.1 \mathrm{~V}$ while the gate voltage was swept forward and backward within a maximum of $\pm 80 \mathrm{~V}$.

\section{Results and discussions}

Before the copper foils undergone the graphene growth process using chemical vapour deposition (CVD), the optical image of the $\mathrm{Cu}$ foil (Fig. 1a, inset) displayed a regular structural pattern that indicated the effects of cold roll processing and polishing. After undergoing the graphene growth process, the surface of these $\mathrm{Cu}$ foils exhibited various dark streaks (Fig. 1a), the 
Please cite as Tan et al. Synthetic Metals 161, 2249 (2011)

cause of which remains unclear. Raman spectroscopy (Reinshaw microRaman at $514 \mathrm{~nm}$ ) was used to examine the as-grown graphene. Raman spectra (Fig. 1b) were taken from the three locations marked in Fig. 1a on the $\mathrm{Cu}$ foil which showed the characteristic single 2D peak at 2710 $\pm 1 \mathrm{~cm}^{-1}$. Figure 1c further shows the derived FWHM of these 2D peaks with Lorenztian curve fittings. The Raman spectra also showed D peak $\left(1367 \mathrm{~cm}^{-1}\right)$ and $\mathrm{G}$ peak $\left(1583 \mathrm{~cm}^{-1}\right)$ though these bands. We did not observe any correlation between the Raman spectra and the above mentioned dark patterns. All these Raman spectra measured were accompanied by a large background signal, thus to better to characterize the material, a transfer procedure is utilized next to transfer the graphene on $\mathrm{Cu}$ foil onto a $\mathrm{SiO}_{2}(\sim 300 \mathrm{~nm}$ thick) substrate using the PMMA transfer method as described in [11]. Figure 2a shows the Raman spectra for the graphene on $\mathrm{SiO}_{2}$ collected at the point indicated (Fig. 2a inset), where both the D (measured at $1348 \mathrm{~cm}^{-1}$ ) and $\mathrm{G}\left(1578 \mathrm{~cm}^{-1}\right)$ peaks can be observed. The 2D Raman peak, Fig.2c, can be fitted to a single Lorentzian line shape centered at $2704 \mathrm{~cm}^{-1}$ and full width half maximum of $37 \mathrm{~cm}^{-1}$, confirming the presence of single layer graphene after transfer [12]. We do note that there are variations in the $I_{2 D}$ to $I_{G}$ ratio over the entire transferred graphene film. This maybe due to the fact that Raman spectrum is influenced by substrate interaction and with a non-uniform adhesion between the graphene film and the $\mathrm{SiO}_{2}$ substrate, a large variation in $\mathrm{I}_{2 \mathrm{D}}$ to $\mathrm{I}_{\mathrm{G}}$ ratio can result [13].

To establish a benchmark for our graphene FET devices, we have employed both top and bottom contact topology graphene FETs using $\mathrm{SiO}_{2}$ as gate dielectric (schematics as shown in Fig. $3 \mathrm{a}$ inset and $3 \mathrm{~b}$ inset respectively) since both top and bottom contact electrode structures are known to strongly influence the electrical performance. Comparing the results for both top and bottom contact $\mathrm{SiO}_{2}$ gate dielectric graphene FETs, there is a marked difference in the drain current $\mathrm{I}_{\mathrm{DS}}$ for the two devices. The device with top contact electrodes had a significantly larger drain current. Using the standard equation for mobility (Eq. 1); 
Please cite as Tan et al. Synthetic Metals 161, 2249 (2011)

$$
\mu=\frac{L}{W} \frac{1}{C} \frac{\partial\left(I_{D S} / V_{D S}\right)}{\partial V_{G S}}
$$

where the gate capacitance $\mathrm{C}$ of $\mathrm{SiO}_{2} \sim 11.5 \mathrm{nF} / \mathrm{cm}^{2}, \mathrm{~L}(\sim 50 \mu \mathrm{m})$ and $\mathrm{W}(\sim 1000 \mu \mathrm{m})$ are dimensions of the device, we determine the mobility of the carriers. In the case of the top contact FET, the calculated typical electron mobility $\sim 1172 \mathrm{~cm}^{2} / \mathrm{Vs}$ for electrons while the calculated hole mobility $\sim 1876 \mathrm{~cm}^{2} / \mathrm{Vs}$, somewhat smaller than the best results reported using $\mathrm{SiC}$ graphitzation [14] and exfoliated HOPG [15]. However, these reported devices were fabricated using top contact electrode topology with a much smaller channel length (typically $<10 \mu \mathrm{m}$ ). In comparison, the bottom contact FET's calculated electron and hole mobilities are $~ 20$ and 24 $\mathrm{cm}^{2} /$ Vs. In bottom contact organic FET, poorer charge injection when compared to top contact FETs is often observed and has been attributed to the higher contact resistance in bottom contacts as well as difficulty in preparing highly ordered organic films on irregular surfaces $[9,10]$. While graphene itself is an ordered structure, irregularity (e.g. surface roughness of underlying support, adsorbates during processing) in topology can have an impact on device performance [16, 17]. Thus we believe similar factors may be at play for a graphene FET as well. From the performance of the above mentioned FETs' performance, we can establish that our CVD grown graphene performance is comparable to other reported material performance in the literature with the top contact electrode topology. For bottom contact electrode topology, to the best of our knowledge, there is no reported result for comparison. With the completion of the benchmarking of our graphene material against published data, we proceed to investigate the performance of graphene as an active channel material for an organic field effect transistor with PVP as the gate dielectric and back gate electrode geometry for our investigation. 
Please cite as Tan et al. Synthetic Metals 161, 2249 (2011)

The electrical $\left(\mathrm{I}_{\mathrm{D}}-\mathrm{V}_{\mathrm{G}}\right)$ characteristics of the graphene FET devices using PVP as polymeric gate dielectrics, were recorded under room temperature in ambient atmosphere or inert atmosphere as indicated. Figure $4 \mathrm{a}$ and $4 \mathrm{~b}$ shows drain current vs back gate voltage for the graphene on PVP FET in ambient environment and after annealing at $80^{\circ} \mathrm{C}$ for 10 minutes in a $\mathrm{N}_{2}$ atmosphere, respectively. We did not observe the neutrality point (i.e. Dirac Point) in the range of gate voltages employed, strongly suggesting that the graphene layer may have some residual impurities that are doping the layer p-type [18]. We did not exceed $\pm 80 \mathrm{~V}$ as the gate leakage becomes significant (> $10 \mathrm{nA}$ range). The gate capacitance of PVP was determined independently using capacitance measurements on a separately prepared PVP film based capacitor structure with Ag electrodes using a Keithley $\mathrm{CV}$ meter to be $\sim 3 \mathrm{nF} / \mathrm{cm}^{2}$.

The hysteresis in the $I_{D}-V_{G}$ characteristic in Fig. 4 a was removed by heating to $80^{\circ} \mathrm{C}$ in a $\mathrm{N}_{2}$ atmosphere glove box with a measured moisture content of $1 \mathrm{ppm}$ or less. There is an improvement in the conductivity and the hysteresis disappeared. But, there is still no evidence of ambipolar transport after heating (Fig. 4b). We did not exceed $80^{\circ} \mathrm{C}$ as we found the gate capacitance changed drastically if heated above $90^{\circ} \mathrm{C}$. The carrier mobility could be found from the variation of the drain current with gate voltage (average slope $2.24 \times 10^{-7} \mathrm{~A} / \mathrm{V}$ ) for a given drain voltage $\left(\mathrm{V}_{\mathrm{D}}=0.1 \mathrm{~V}\right)$ from the previous equation (1).

From the previously measured value of $\mathrm{C}_{\mathrm{G}}=3 \mathrm{nF} / \mathrm{cm}^{2}$ we find that the hole mobility is 37 $\mathrm{cm}^{2} / \mathrm{Vs}$ for the sample after heating. This result is comparable to some of the best reported organic semiconductor based FET of $\sim 10 \mathrm{~cm}^{2} /$ Vs $[19,20]$. For the absence of ambipolar transport characteristics that is observed in $\mathrm{SiO}_{2}$ gate dielectric based graphene FET, it is possible that the presence of silanol groups, which are part of molecular structure in the PVP polymer, could both have reduced graphene's intrinsic mobility and be responsible for the absence of ambipolar conduction. It is known that in carbon nanotube field effect transistors the absence of ambipolar 
Please cite as Tan et al. Synthetic Metals 161, 2249 (2011)

conduction was attributed to the effects of silanol groups [23]. Incidentally, a similar phenomena is also reported for organic transistors [21, 22]. As mentioned surface roughness also affects mobility, thus AFM measurement was performed on our PVP film and we found that the rms roughness $\sim 3.6 \mathrm{~nm}$ is slightly rougher than measured $\mathrm{SiO}_{2}$ surface roughness $\sim 2 \mathrm{~nm}$ rms (wafer supplied by Si-Mat).

In summary, we have demonstrated field effect in CVD grown graphene using $\mathrm{Cu}$ foil for growth and subsequently transferred to a back gate bottom contact transistor arrangement using poly-4-vinyl-phenol as an alternative to oxide based insulators. In our study, we used a simple, inexpensive fabrication method that is fully compatible with present large scale manufacture of organic devices, to demonstrate a field effect hole mobility of $37 \mathrm{~cm}^{2} / \mathrm{Vs}$. This mobility despite being lower than that found in exfoliated graphene (on $\mathrm{SiO}_{2}$ ) demonstrates the possibility of a graphene based all carbon transistors for large area electronics.

\section{Acknowledgements:}

N. Nismy and R. Sporea for assisting in some of the FETs' fabrication and measurements. 
Please cite as Tan et al. Synthetic Metals 161, 2249 (2011)

\section{References}

1 K. S. Novoselov, A. K. Geim, S. V. Morozov, D. Jiang, Y. Zhang, S. V. Dubonos, I. V. Grigorieva, and A. A. Firsov, Science 306 (2004) 666.

2 A. H. Castro Neto, F. Guinea, N. M. R. Peres, K. S. Novoselov and A. K. Geim, Rev, Mod. Phys. 81 (2009) 109.

3 S. Adam, S., E. H. Hwang, V. M. Galitski, and S. das Sarma, Proc. Natl. Acad. Sci. U.S.A. 104 (2007) 18392.

4 X. Li, W. Cai, J. An, S. Kim, J. Nah, D. Yang, R. Piner, A. Velamakanni, I. Jung, E. Tutuc, S. K. Banerjee, L. Colombo and R. S. Ruoff, Science 324 (2009) 1312.

5 S. Bae, H. Kim, Y. Lee, X. Xu, J-S Park, Y. Zheng, J. Balakrishnan, T. Lei, H. R Kim, Y.I Song, Y-J Kim, K.S. Kim, B. Özyilmaz, J-H Ahn, B. H Hong and Sumio Iijima, Nature Nano. 5 (2010) 574.

6 H. S. Byun, Y. X. Xu and C. K. Song, Thin Solid films, 493 (2005) 278.

7 J. W. Kim, J. W. Jeong, H. D. Cho, C. H. Lee, S. O. Kim, S. K. Kwon and Y. T. Hong, J. Phys. D: Appl. Phys. 42 (2009) 115107.

8 A. Facchetti, M. H. Yoon, and T. J. Marks, Adv. Mater. 17 (2005) 1705-1725.

9 Zhenan Bao and Jason Locklin, Organic Field-Effect Transistors, CRC press, 2007.

10 A. R. Murphy, Jean M. J. Frechet, Chem. Rev. 1066 (2007).

11 A. Reina, X. Jia, J. Ho, D. Nezich, H. Son, V. Bulovic, M. S. Dresselhaus and J. Kong, NanoLett. 9 (2008) 30.

12 C. Ferrari, J. C. Meyer, V. Scardaci, C. Casiraghi, M. Lazzeri, F. Mauri, S. Piscanec, D. Jiang, K. S. Novoselov, S. Roth, and A. K. Geim, Phys. Rev. Lett. 97 (2006) 187601.

13 S. Berciaud, S. Ryu, L. E. Brus, T. F. Heinz, NanoLett. 9 (2009) 346.

14 J. Kedzierski, P. L. Hsu, P. Healey, P. Wyatt, C. L. Keast, M. Sprinkle, C. Berger, and W.A. de Heer, IEEE Trans. Electron Device 55, 8 (2008) 2078-2085.

15 A. K. Geim, K.S. Novoselov, Nat. Mater. 6 (2007) 3.

16 J. H. Chen, C. Jang, M. Ishigami, S. Xiao, W. G. Cullen, E. D. Williams, M. S. Fuhrer, Solid State Commum. 149 (2009) 1080.

17 S. M. I. Katsnelson and A. K. Geim, Phil. Trans. R. Soc. A 366 (2008)195-204. 
Please cite as Tan et al. Synthetic Metals 161, 2249 (2011)

18 F. Schedin, A. K. Geim, K. S. Novoselov, S. V. Morozov, E. W. Hill, P. Blake, M. I. Katsnelson, Nat. Mater. 6 (2007) 652.

19 L. B. Roberson, J. Kowalik, L. M. Tolbert, C. Kloc, R. Zeis, X. Chi , R. Fleming, C. Wilkins, J. Am. Chem. Soc.127 (2005) 3069.

20 V. Podzorov, S. E. Sysoev, E. Loginova, V. M. Pudalov, M. E. Gershenson, Appl. Phys. Lett. 83 (2003) 3504.

21 T. B. Singh, F. Meghdadi, S. Gunes, N. Marjanovic, G. Horowitz, P. Lang, S. Bauer, N. S. Sariciftci, Adv. Mater. 17 (2005) 2315.

22 L. L. Chua, J. Zaumseil, J. F. Chang, E. C. W. Ou, P. K. H. Ho, H. Sirringhaus, R. H. Friend, Nature 434 (2005) 194.

23 C. M. Aguirre, P. L. Levesque, M. Paillet, F. Lapointe, B. C. St-Antonine, P. Desjardins, R. Martel, Adv. Mater. 21 (2009) 3087. 
Please cite as Tan et al. Synthetic Metals 161, 2249 (2011)
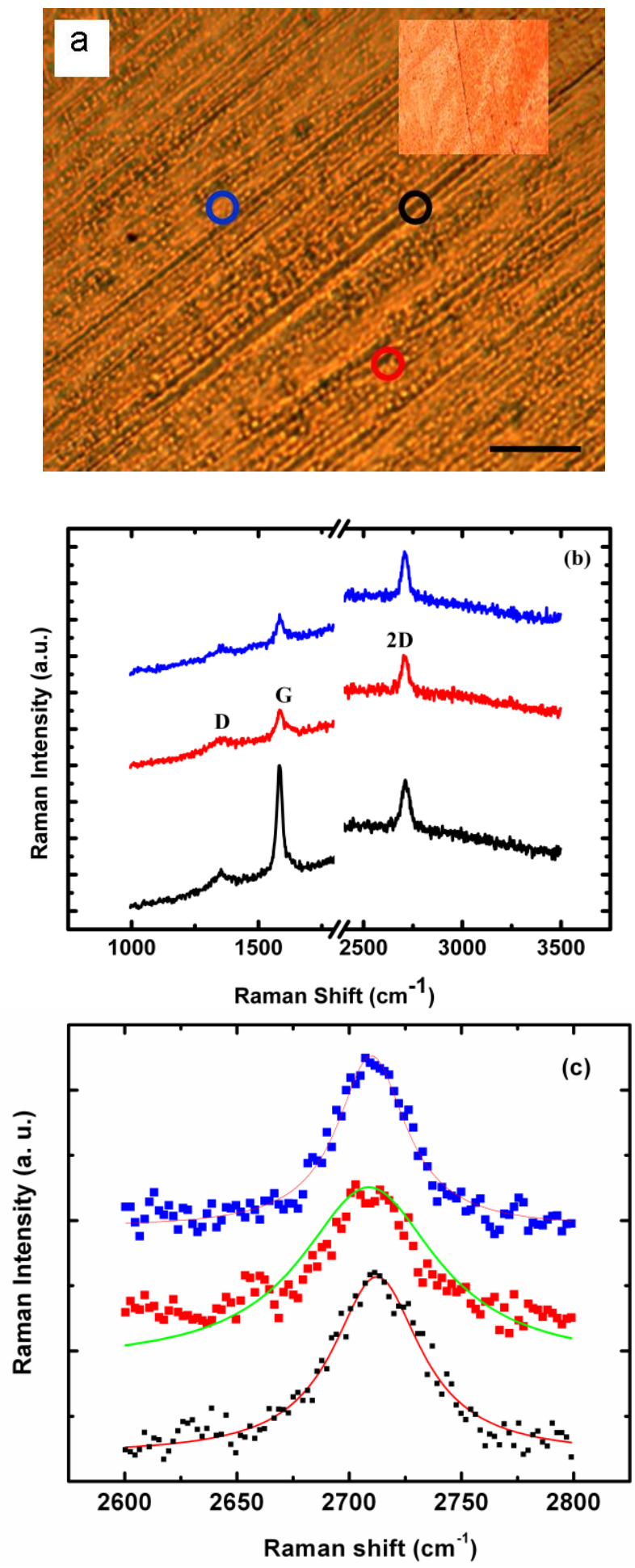

Figure 1: (a) Optical image of graphene as grown on $\mathrm{Cu}$, inset is image of $\mathrm{Cu}$ before processing. The black bar is $50 \mu \mathrm{m}$. (b) Raman spectra of the three corresponding points (as highlighted in colors in the figure 1a) indicating the D, G and 2D peaks. Note the large background signal. (c) Raman 2D peak spectra and their Lorentzian curve fittings at three corresponding points on the optical image shown previously in figure 1a. Blue circle $\sim 2709 \mathrm{~cm}^{-1} \mathrm{FWHM} \sim 37 \mathrm{~cm}^{-1}$. Red circle $\sim 2708 \mathrm{~cm}^{-1}$, FWHM $\sim 64 \mathrm{~cm}^{-1}$. Black circle $\sim 2712 \mathrm{~cm}^{-1}$ FWHM $\sim 47 \mathrm{~cm}^{-1}$. 
Please cite as Tan et al. Synthetic Metals 161, 2249 (2011)
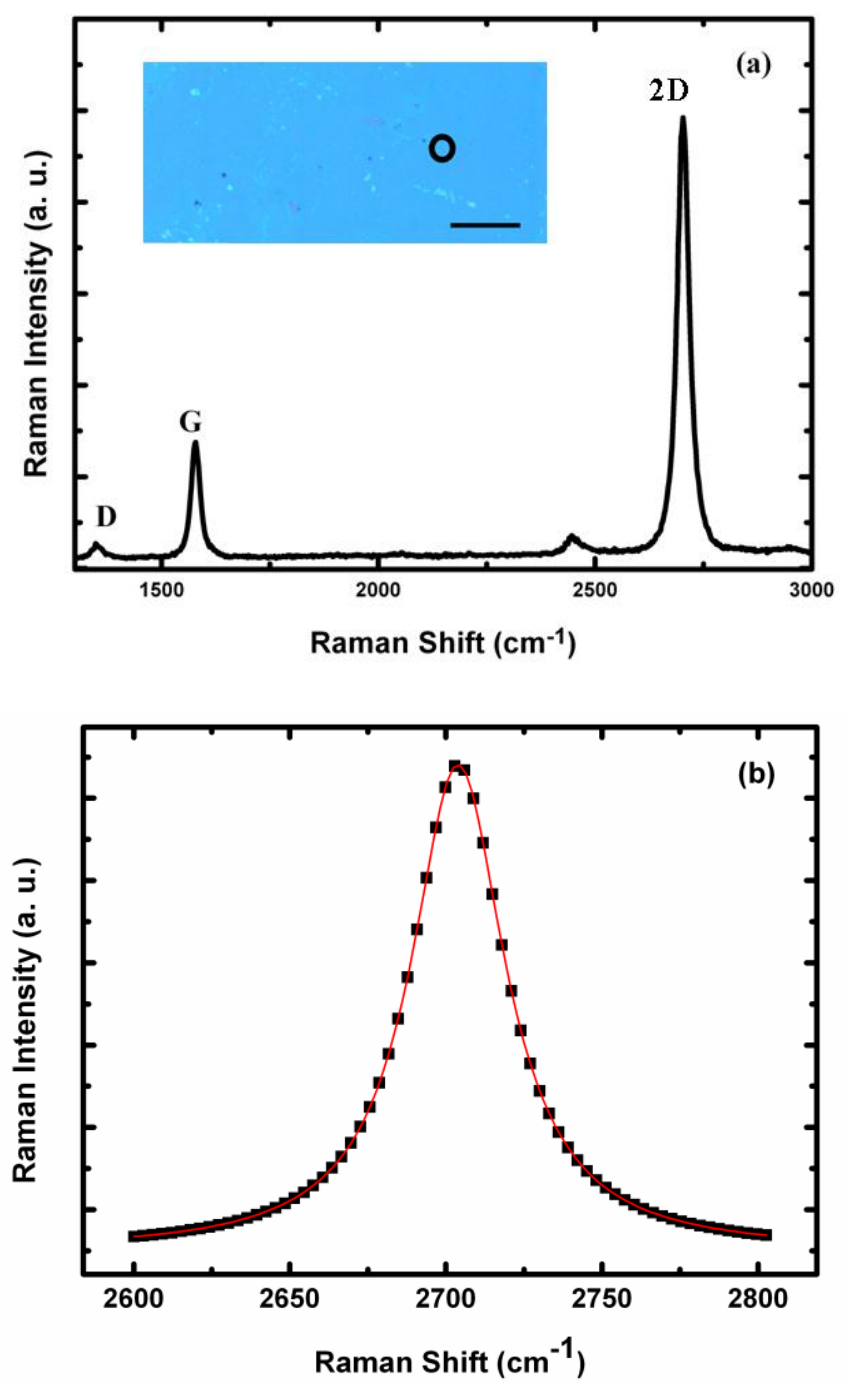

Figure 2: (a) Raman spectrum of graphene after transferring from copper onto $\mathrm{SiO} 2$ substrate (thickness $~ 300 \mathrm{~nm}$ ) as highlighted by the circle in the optical image of the graphene (as shown in inset of figure $2 \mathrm{a}$ ). The black bar is $50 \mu \mathrm{m}$. The spectrum indicated various $\mathrm{D}, \mathrm{G}$ and $2 \mathrm{D}$ peaks. (b) Raman 2D peak spectra and the Lorentzian curve fitting at the same corresponding point on the optical image shown previously in figure $2 \mathrm{a}$ inset, indicating the presence of single layer graphene as transferred onto the $\mathrm{SiO}_{2}$ surface.. 
Please cite as Tan et al. Synthetic Metals 161, 2249 (2011)
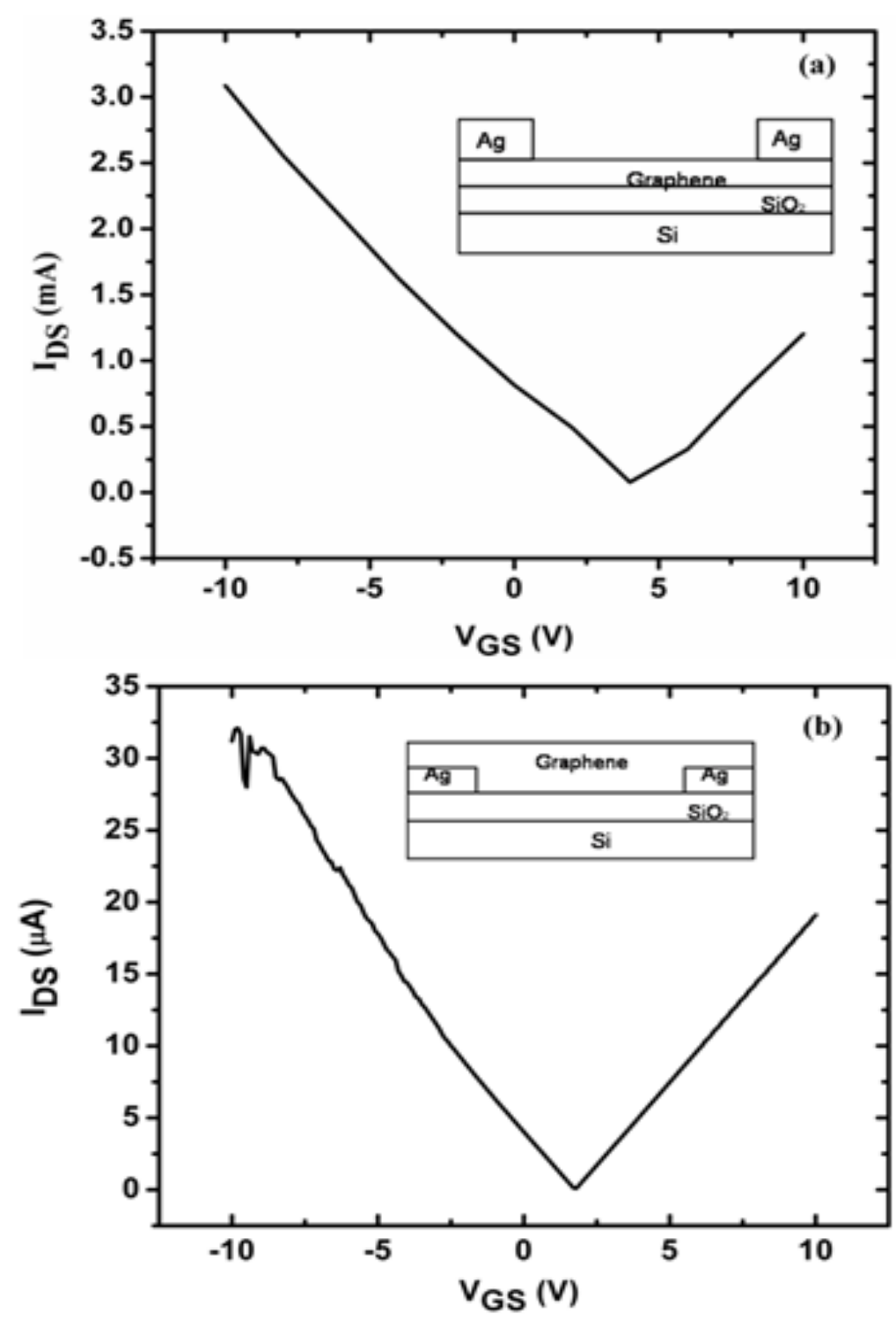

Figure 3: Measurements drain current versus gate $\left(I_{D}-V_{G S}\right)$ sweeping voltage of silicon back gate graphene FETs using 300nm $\mathrm{SiO}_{2}$ as gate dielectric and their respective schematics for (a) top contact and (b) bottom contact FET. All FET are back gate by design. Measurement was done in air ambient at room temperature. All drain source voltage is biased at $\mathrm{V}_{\mathrm{DS}}=0.5 \mathrm{~V}$. 
Please cite as Tan et al. Synthetic Metals 161, 2249 (2011)
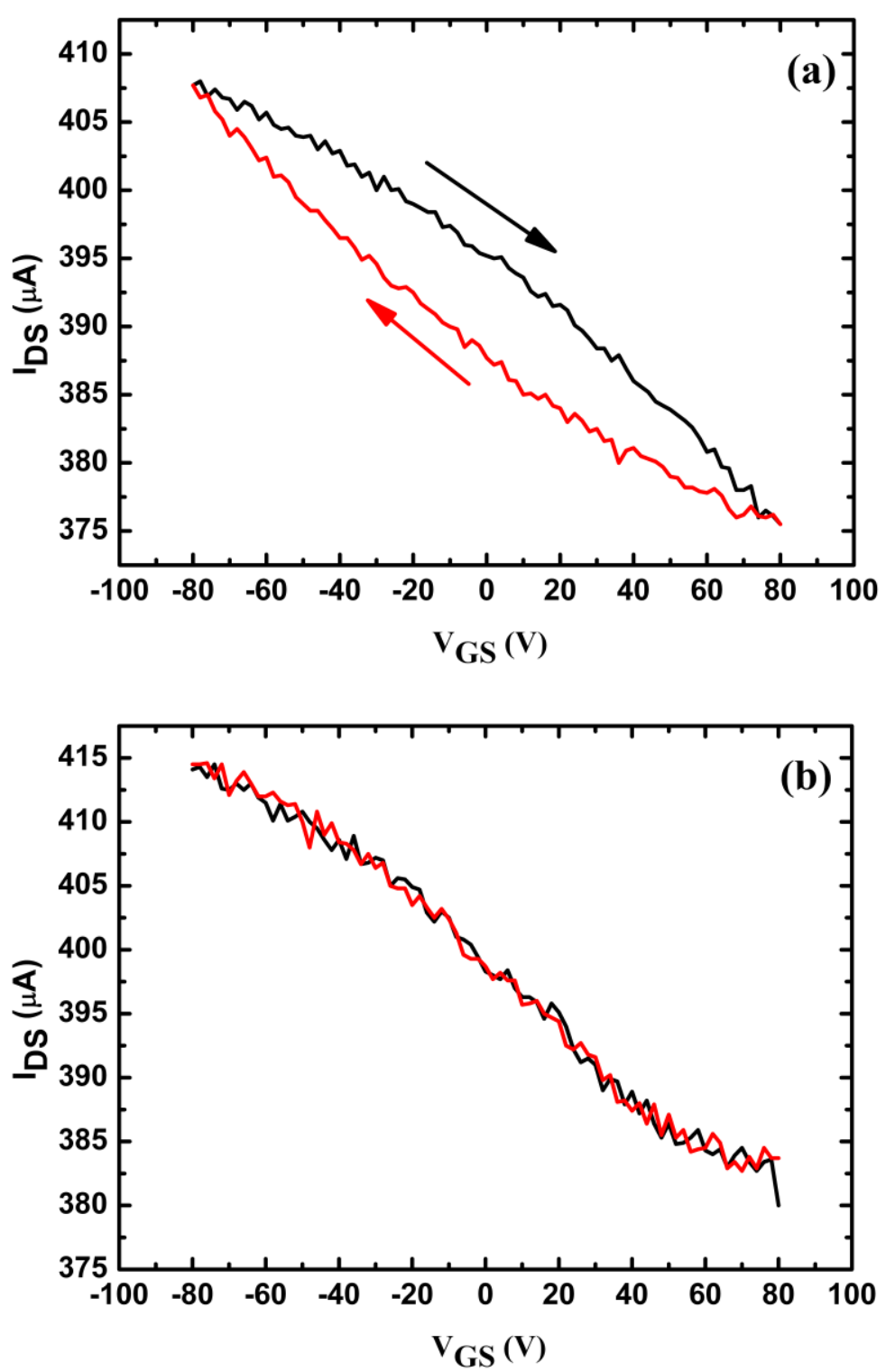

Figure 4: (a) Drain current versus gate sweeping voltage of back-gate, bottom contact graphene FET using PVP polymer $(1 \mu \mathrm{m})$ as gate dielectric on a Corning glass substrate. The measurement was done in air ambient at room temperature and (b) measured in an inert ambient $\left(\mathrm{N}_{2}\right)$ chamber after heating the device for $10 \mathrm{~min}$ at $80^{\circ} \mathrm{C}$ under $\mathrm{N}_{2}$ atmosphere. All drain source voltage is biased at $\mathrm{V}_{\mathrm{DS}}=0.1 \mathrm{~V}$. 